\title{
"A scuola di Astroparticelle": a synergy between school education and scientific research
}

\author{
Roberta Colalillo ${ }^{a, b, *}$ and Carla Aramo ${ }^{a}$ on behalf of the OCRA Collaboration \\ (a complete list of authors can be found at the end of the proceedings) \\ ${ }^{a}$ INFN, Sezione di Napoli, Via Cintia, 80126 Napoli, Italy \\ ${ }^{b}$ Università degli Studi di Napoli "Federico II", Dipartimento di Fisica “E. Pancini”, Via Cintia, \\ 80126 Napoli, Italy \\ E-mail: colalillo@na.infn.it
}

The outreach program "A scuola di Astroparticelle" was proposed in 2016 by the National Institute for Nuclear Physics (INFN - Napoli Division) in collaboration with the Physics Department "Ettore Pancini" of the Federico II University in Napoli, CNR-SPIN and CNR-ISASI Institutes. Its main goal is to engage teachers and students of High Schools in astroparticle physics projects.

For the third edition (2018/19), the activities, which are also part of the Italian Educational Program PCTO - "Percorsi per le Competenze Trasversali e per l'Orientamento", involved 18 schools for a total of 21 projects on several topics. Some projects were strictly related to astroparticles as cosmic rays, while others were more technical, as the development of particle detectors, or crossdisciplinary projects. Students worked for the entire school year and prepared materials for the final event. More than 600 students attended the event and presented their work to a jury with a poster and an oral presentation in plenary sessions. Since 2018, the program is part of OCRA Outreach Cosmic Ray Activities - a national outreach project of INFN with the aim of collecting, within a common framework, the numerous outreach activities in cosmic-ray field carried out at the local level.

The fourth edition (2019-20), in spite of the difficult situation due to the COVID-19 pandemic, has also seen the participation of 22 schools that carried out part of the activities in an online format. The project realized using the open data of the Pierre Auger Observatory will be presented in detail.

$37^{\text {th }}$ International Cosmic Ray Conference (ICRC 2021)

July 12th-23rd, 2021

Online - Berlin, Germany

\footnotetext{
*Presenter
} 


\section{Introduction}

The mission of the National Institute for Nuclear Physics (INFN) includes, in addition to research, the transfer of acquired knowledge to society. By this we mean both the sharing of know-how in the form of training and technology transfer, and the spreading of scientific culture. According to this second aspect of the mission, the outreach program "A scuola di Astroparticelle" [1,2] was born with the idea of engaging teachers and students of High Schools in astroparticle physics projects, which are an important part of the INFN reasearch program, but also in the development of particle detectors, in astrophysics, nuclear, optics, biomedical and environmental prevention activities. The structure of "A scuola di astroparticelle" fully meets the requirements of MIUR (Ministero per l'Istruzione, Università e Ricerca - italian authority for education) for the Italian Educational Program PCTO - "Percorsi per le Competenze Trasversali e per l'Orientamento" [3]: ability to interact and work with others, problem solving skills, creativity, critical thinking, awareness, resilience and ability to identify available forms of guidance and support to address the complexity and uncertainty of changes.

Since 2018, the program is part of OCRA (Outreach Cosmic Ray Activities), the INFN program [5] that coordinates, at the national level, the scientific outreach activities in the field of cosmic rays, and that allows students and teachers to get closer to the world of astroparticle physics in more than 21 cities.

In the following sections, we will describe the several phases of "A scuola di Astroparticelle" which contribute to student education and, in particular, the activities carried out with the open data of the Pierre Auger Observatory.

\section{Discovering "A scuola di Astroparticelle"}

The activities of "A scuola di Astroparticelle" are organized in 5 days of 8 hours, for a total of 40 hours of work. On the first day, students encounter for the first time the cosmic radiation, invisible to human eyes, which continuously passes through our bodies. They visit the Toledo metro station in Napoli where, in 2014, a telescope was installed to detect cosmic muons [4]. When a muon passes through the telescope, a light track is displayed in two-dimensions. The telescope is coupled to a multimedia touchscreen that allows the acquisition of data to reconstruct the direction of the muons and display their tracks in three-dimensions on the screen. In the afternoon, they vist the university and the laboratories accompanied by the researchers which will be their tutors for the entire course. This first approach allows students to begin to familiarize with the world of research and they get to know what it means to make a measurement. On the second day, the tutor introduces students to the topic that will be the core of their PCTO. In this phase, the interaction with the tutor is fundamental for the students to be able to enter in this new world. The final part of the day is dedicated to the organization of the upcoming activities, which can be laboratory activities, research on the territory, data analysis. The first phases will be carried out all together with the supervision of the tutor, then the students will be split into small groups. In this way, they will be able to unleash their creativity and autonomously develop their working techniques. Furthermore, they will have to deal with the opportunities and challanges related with working in a team. On the third day, students will have to develop their research and start the preparation for the final event, 


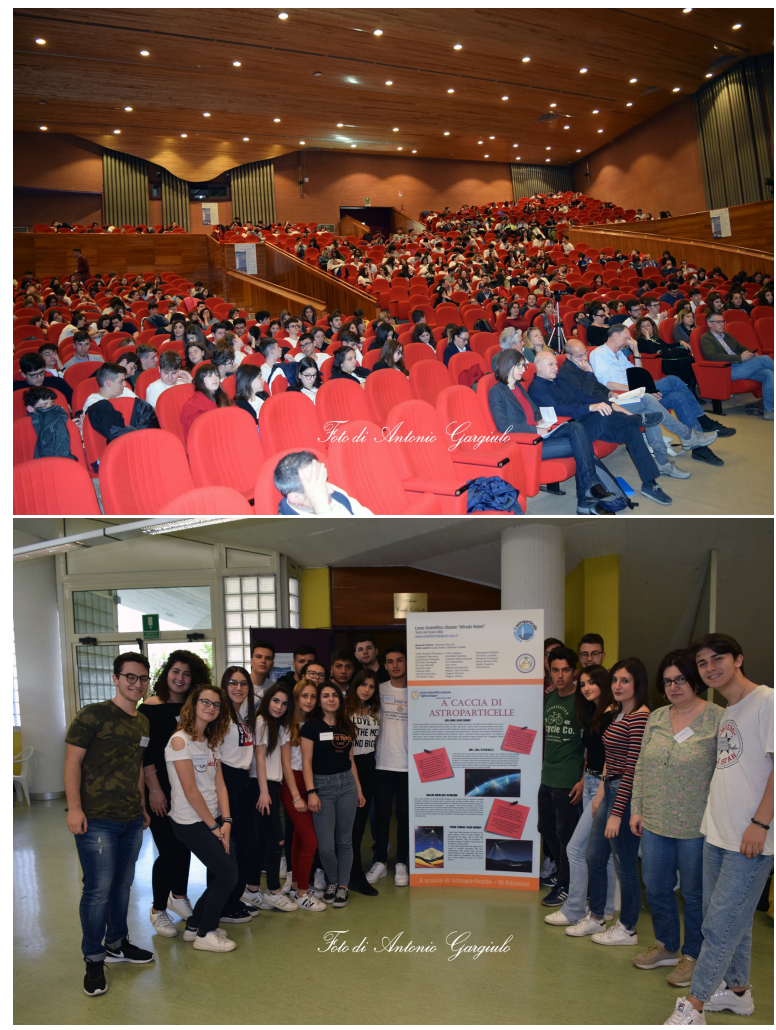

Figure 1: The final event of the third edition (2018/2019) of "A scuola di Astroparticelle". Top: The audience and the jury members that listen student presentation. Bottom: A class of the Scientific High School "Alfred Nobel" in Torre del Greco (NA) that reproduced the elongation rate to study the mass composition of ultra-high energy cosmic rays with the data of the Pierre Auger Observatory.

which will take the form of a conference. Students will write their proceeding, will prepare a talk and a poster that they will present to their colleagues and to the jury members. Two pictures of the final event of the third edition (2018/2019), that involved 18 schools for a total of 21 projects, are shown in Fig. 1.

\section{The fourth edition: a special online edition}

Due to the COVID-19 pandemic, the 2019/20 edition was suspended and was resumed in the school year 2020/21 in online mode. Each tutor adapted the activity to allow students to meet online and work together as also researchers are doing in this very particular period of our life. This special edition involved 10 schools for a total of 18 projects. Also the final event was carried out online using the platform 'Zoom' [6]. Students took turn in exhibiting their works during a whole morning and were evaluated by the jury, that had the arduous task of selecting the winning work of the fourth edition. While waiting to receive the response, OCRA activities were presented. In spite of many difficulties due to the pandemic, students showed great maturity, as well as a lot of enthusiasm, and the result can be appreciated on the OCRA Indico page where it is also possible to 


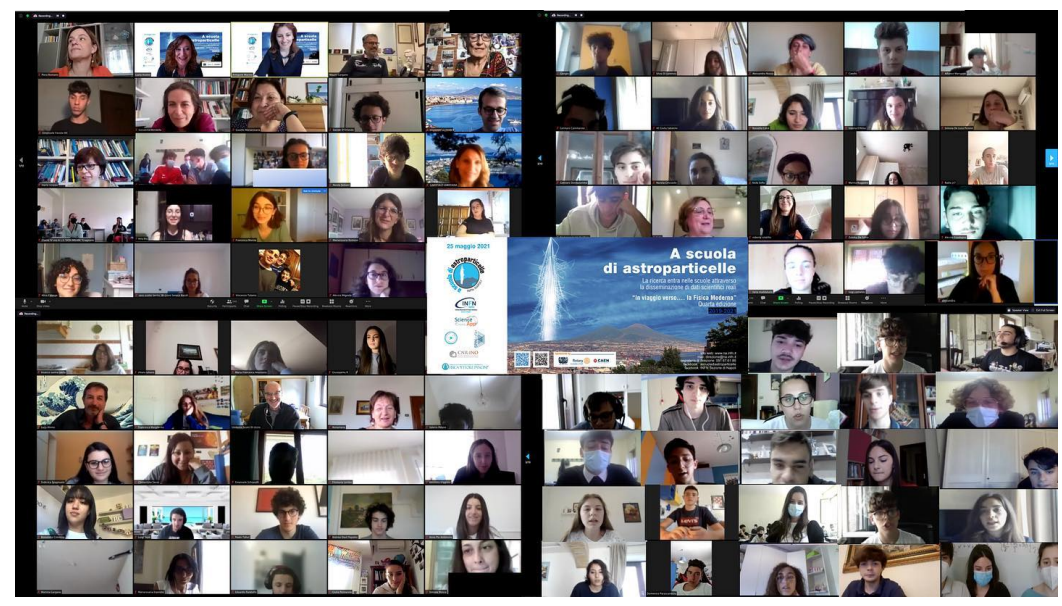

Figure 2: Students, teachers and tutors in Zoom connection during the final event of the special fourth edition held on May 25, 2021.

follow the complete registration of the event. In Fig. 2, a screenshot with a part of the 300 students and tutors who were the 'soul' of the fourth edition of "A scuola di Astroparticelle" is shown.

\section{Activities with the public data of the Pierre Auger Observatory}

The Pierre Auger Observatory [8] is the largest detector in the world to study the extensive air showers produced by the interaction of ultra-high energy cosmic rays with atmospheric molecules. It is located on a high-altitude plain in Argentina and consists of a fluorescence detector (FD) that studies the longitudinal development of the shower and a surface detector (SD) that samples the distribution of the shower particles at the ground. The surface detector is an array of 1600 waterCherenkov particle detector stations spread over $3000 \mathrm{~km}^{2}$ on a $1500 \mathrm{~m}$ triangular grid, overlooked by 24 air-fluorescence telescopes. These telescopes are organized in four sites, 6 for each site, called Los Leones, Los Morados, Loma Amarilla, and Coihueco.

The Pierre Auger Collaboration has recently released $10 \%$ of the data recorded with the two detectors [7]. All datasets have a unique DOI that you are requested to cite in any applications or publications. The current release should be cited as: Pierre Auger Collaboration (2021), Auger Open Data release 1-2021, DOI:10.5281/zenodo.4487613. The purpouse of the release is the reuse of data by a wide community including professional scientists, in educational and outreach initiatives, and by citizen scientists. We used these data for "A scuola di Astroparticelle". Two fifth grade classes of Scientific High School reproduced the measurement of the energy spectrum of vertical cosmic rays (zenith $<60$ degrees) detected with the Pierre Auger surface detector [10].

Before analyzing data, we explained to the students the cosmic rays: what we already know about these mysterious particles and the questions still open 100 years after their discovery. The Pierre Auger Collaboration is composed by about 400 scientists, engineers, technicians and students from more than 90 institutions in 18 countries. Introducing students to the dynamics of the collaboration is also a way to let them know the importance of working in team. The main goals of the collaboration are the determination of the mass composition of the ultra-high energy cosmic rays, the measurement of the energy spectrum and the study of the cosmic-ray arrival direction. Students 

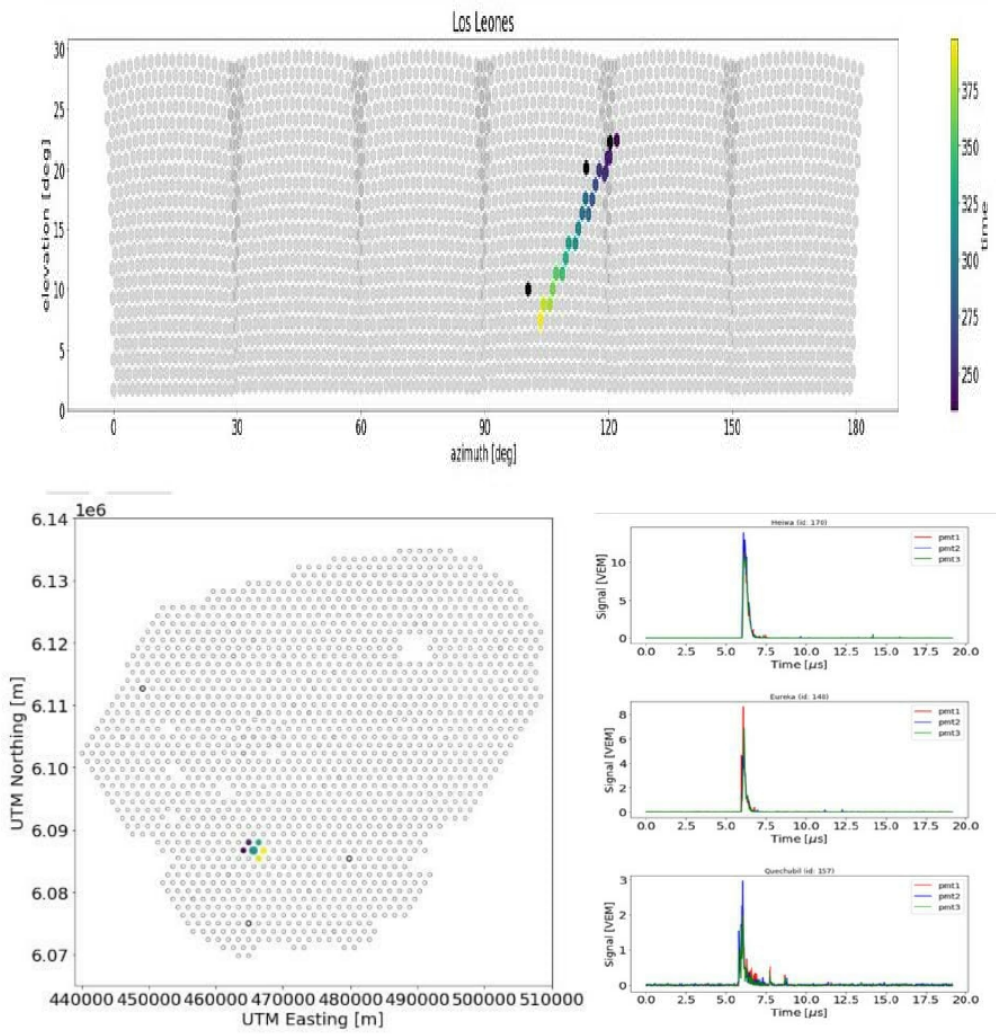

Figure 3: An hybrid event detected at the Pierre Auger Observatory. Top: trace of the shower in the camera of the fluoresce telescope in Los Leones. Bottom: map of the triggered SD stations on the left; the signals collected in some triggered stations on the right.

understood what is an extensive air shower and how it is possible to obtain information on the cosmic ray that produced a shower, from its imprint in the detectors. They studied the differences between the hybrid events, which are showers detected by at least one site of the fluorescence detector and at least one station of the surface station, and the events detected only by the surface detector. Using Python notebooks provided by the collaboration [9], they analyzed an hybrid shower. In Fig. 3, we can see the trace of the shower in the fluorescence telescope camera, the footprint of the event at the ground and the signal in some triggered stations. For the measurement of the energy spectrum, instead, students used the Python notebooks [13] available on the OCRA webpage [12]. A series of online tools and interactive laboratories on cosmic rays were developed during the first lockdown in Italy, in the spring of 2020, to support High School students to prepare their thesis work for their final exam [14].

The energy spectrum is made by counting the number of observed showers in differential energy bins and dividing this number by the exposure. The exposure is a normalization factor that takes into account the time the detector has been taking data, the surface available for measurements and the portion of the sky observed. During the last edition, students reproduced the SD spectrum represented with gray dots in the plot on the left of Fig. 4. The official energy spectrum is calculated with a bin size equal to 0.1 logarithm of the energy. In our case, we have only the $10 \%$ of the Auger data, and with this bin size, we have many statistic fluctuations. Students reproduced the vertical 

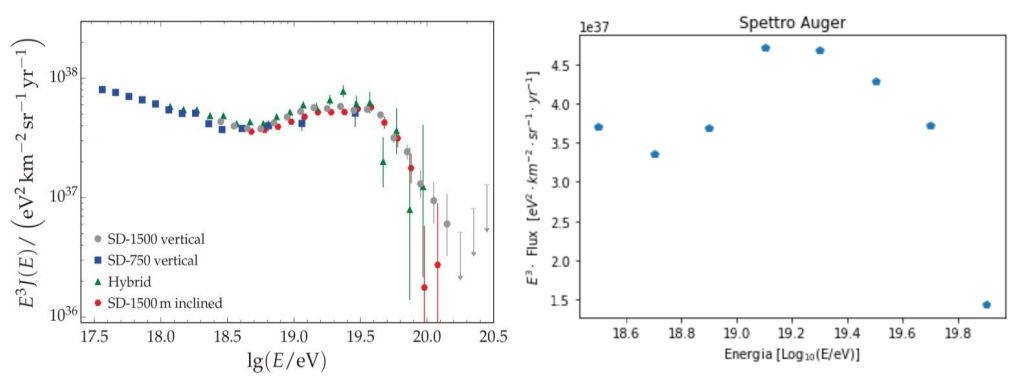

Figure 4: Left: The energy spectra published by the Pierre Auger Collaboration. The spectrum obtained from the vertical showers (zenith $<60$ degrees) detected with the SD is represented in gray. Right: The energy spectrum of vertical cosmic rays obtained by students using the $10 \%$ of the SD data of the Pierre Auger Observatory.

spectrum with bin size $0.1 \log (\mathrm{E} / \mathrm{eV})$ and $0.2 \log (\mathrm{E} / \mathrm{eV})$. They compared the two trends and chose the spectrum with bin size $0.2 \log (\mathrm{E} / \mathrm{eV})$ because the fluctuations are minor and the features of the spectrum can be better observed. On the right of Fig. 4, the SD energy spectrum of vertical cosmic rays is shown and it is compared with the published one shown in gray on the right. The two spectra are comparable but we have to remember that students worked with a small statistic.

\section{Conclusions}

The students were enthusiastic about this experience which allowed them to approach the world of research, perceived as distant and hostile by most of them until that moment. In addition, they used the newly acquired knowledge for their final exam and the work done was highly appreciated by the jury.

In this fourth edition of "A scuola di Astroparticelle", we learnt how to improve this project together with students, how to get students involved more and more, and what tools to provide them. Moreover, we tested that it is a valid tool for educational and personal growth of young students. In the next years, we will continue to offer "A scuola di Astroparticelle" as PCTO and to expand the available research paths.

\section{References}

[1] C. Aramo et al., PoS EPS-HEP2017 (2018) 549.

[2] https://www.facebook.com/ascuoladiastroparticelle.

[3] MIUR - PCTO.

[4] F. Arneodo et al., Nucl. Instrum. Meth. A 799 (2015) 166-171.

[5] C. Aramo and S. Hemmer [for the OCRA Coll.], Proc. 36th Int. Cosmic Ray Conf., Madison, WI, USA (2019), PoS(ICRC2019)173.

[6] C. Aramo, SIF prima pagina, N.90, 1324, Giugno 2021.

[7] V. Scherini [for the Pierre Auger Coll.], these proceedings.

[8] Pierre Auger Coll., Nucl. Instrum. Meth. A 798 (2015) 172-213, [1502 . 01323].

[9] https://opendata.auger.org/analysis.php

[10] F. Fenu [for the Pierre Auger Coll.], Proc. 35th Int. Cosmic Ray Conf., Busan, Korea (2017), PoS(ICRC2017)486.

[11] https://opendata.auger.org/analysis.php

[12] https://web.infn.it/OCRA/. 
[13] Google Colab - spectrum python scripts.

[14] C. Aramo [for the OCRA Coll.], these proceedings. 


\section{The OCRA Collaboration}

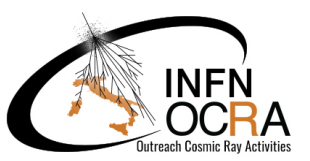

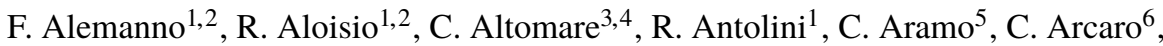
F.C.T. Barbato ${ }^{1,2}$, M. Battaglieri ${ }^{7}$, M. Battisti ${ }^{8,9}$, A. Bau' ${ }^{10,11}$, V.E. Bellinzona ${ }^{12}$, P. Bernardini ${ }^{13,14}$, A. Bersani ${ }^{7}$, M. Bertaina ${ }^{8,9}$, A. Berti ${ }^{8}$, B. Bertucci ${ }^{15,16}$, F. Bisconti ${ }^{8,9}$, E. Bissaldi ${ }^{3,4}$, V. Bocci ${ }^{17}$, M. Boezio ${ }^{18,19}$, D. Boncioli ${ }^{1,20}$, M. Bond $i^{7}$, L. Bonechi $^{21}$, R. Bonino ${ }^{8,9}$, G. Bonnoli ${ }^{22}$, V. Bonvicini ${ }^{18}$, E. Bossini ${ }^{22,23}$, B. Bottino ${ }^{7,24}$, M. Buscemi ${ }^{25}$, B. Caccianiga ${ }^{26}$, L. Caccianiga ${ }^{26}$, A. Candela ${ }^{1}$, A. Capone ${ }^{17,27}$, M. Cariello ${ }^{7}$, R. Caruso ${ }^{25,28}$, G. Cataldi ${ }^{13}$, G. Chiodi ${ }^{17}$, G. Chiodini ${ }^{13}$, R. Colalillo ${ }^{5,29}$, M.R. Coluccia ${ }^{13}$, F. Convenga ${ }^{13,14}$, S. Copello ${ }^{7,30}$, M. Corosu ${ }^{7}$, D. D’Urso ${ }^{31,32}$, F. Dal Corso ${ }^{6}$, S. Davini ${ }^{7}$, M. De Deo ${ }^{1}$, S. De Gateano ${ }^{3,4}$, M. De Laurentis ${ }^{5,29}$, I. De Mitri ${ }^{1,2}$, F. De Palma ${ }^{13,14}$, E. De Vito ${ }^{13,14}$, D. Dell'Aquila ${ }^{31,32}$, D. Depaoli ${ }^{8,9}$, A. Di Luca ${ }^{12}$, F. Di Pierro ${ }^{8}$, B. Di Ruzza $^{12}$, M. Di Santo ${ }^{1,2}$, G. Di Sciascio ${ }^{33}$, L. Di Venere ${ }^{3,4}$, F. Dimiccoli12, K. Dimitrios1, 2, F. Donnini15, M. Doro ${ }^{6,34}$, M. Duranti ${ }^{15}$, C. Evoli ${ }^{1,2}$, F. Fenu ${ }^{8}$, F. Fontanelli ${ }^{7,30}$, P. Fusco ${ }^{3,4}$, F. Gargano ${ }^{3}$, M. Gervasi ${ }^{10,11}$, A. Giampaoli ${ }^{1}$, N. Giglietto ${ }^{3,4}$, F. Giordano ${ }^{3,4}$, I. Gnesi ${ }^{35,36}$, S. Gonzi ${ }^{21}$, D. Grandi ${ }^{10,11}$, M. Graziani ${ }^{15,16}$, S. Hemmer ${ }^{6}$, F. Iacoangeli ${ }^{17}$, A. Insolia ${ }^{25,28}$, S. Iovenitti ${ }^{26,37}$, V. Ippolito ${ }^{17}$, G. La Vacca ${ }^{10,11}$, G. La Verde ${ }^{5,29}$, E. Leonora ${ }^{25}$, S. Levorato ${ }^{18}$, D. Liguori ${ }^{35,38}$, P. Lipari ${ }^{17}$, F. Longo ${ }^{8,39}$, F. Loparco ${ }^{3,4}$, R. Lopez Coto ${ }^{6}$, S. Loporchio $^{3}$, A. Marino ${ }^{5,40}$, G. Marsella ${ }^{25,41}$, D. Martello ${ }^{13,14}$, M.N. Mazziotta ${ }^{3}$, A. Menegolli ${ }^{42,43}$, S. Miozzi ${ }^{33}$, H. Miyamoto ${ }^{8}$, E. Mocchiutti ${ }^{18}$, S. Morganti ${ }^{17}$, F. Morsani ${ }^{22}$, R. Munini ${ }^{18,19}$, R. Mussa ${ }^{8}$, F. Nozzoli ${ }^{12}$, A. Nucita ${ }^{13,14}$, G.C. Organtini ${ }^{17,27}$, G. Ottonello ${ }^{7}$, F. Pantaleo ${ }^{3,4}$, R. Paoletti ${ }^{22}$, F. Parodi ${ }^{7}$, L. Perrone ${ }^{13,14}$, L. Pesenti ${ }^{10,11}$, S. Petrera ${ }^{1,2}$, C. Petronio ${ }^{35,44}$, R. Pillera ${ }^{3,4}$, F. Pilo ${ }^{22}$, C. Pizzolotto ${ }^{18}$, E. Prandini 6,34 , M. Pugliese ${ }^{5,29}$, S. Rain $\grave{o}^{3,4}$, N. Randazzo ${ }^{25}$, R. Rando6,34,45, L. Recchia ${ }^{17}$, E. Ricci ${ }^{12}$, M. Rinaudo ${ }^{8,9}$, V. Rizi ${ }^{1,20}$, N. Rossi ${ }^{1}$, D. Rozza ${ }^{31,32}$, F. Salamida ${ }^{1,20}$, P. Savina ${ }^{13,14}$, V. Scherini ${ }^{13.14}$, M. Schioppa ${ }^{35,46}$, V. Scotti ${ }^{5,29}$, D. Serini ${ }^{3}$, V. Sipala ${ }^{31,32}$, A. Surdo ${ }^{13,14}$, A. Tiberio ${ }^{21}$, N. Tomassetti ${ }^{15,16}$, C. Tomei ${ }^{17}$, I. Tosta E Melo ${ }^{31,32}$, P. Turco ${ }^{35,46}$, E. Vannuccini ${ }^{21}$, V. Vecchiotti ${ }^{1,2}$, I. Veronesi ${ }^{5,47}$, G. Zampa ${ }^{18}$

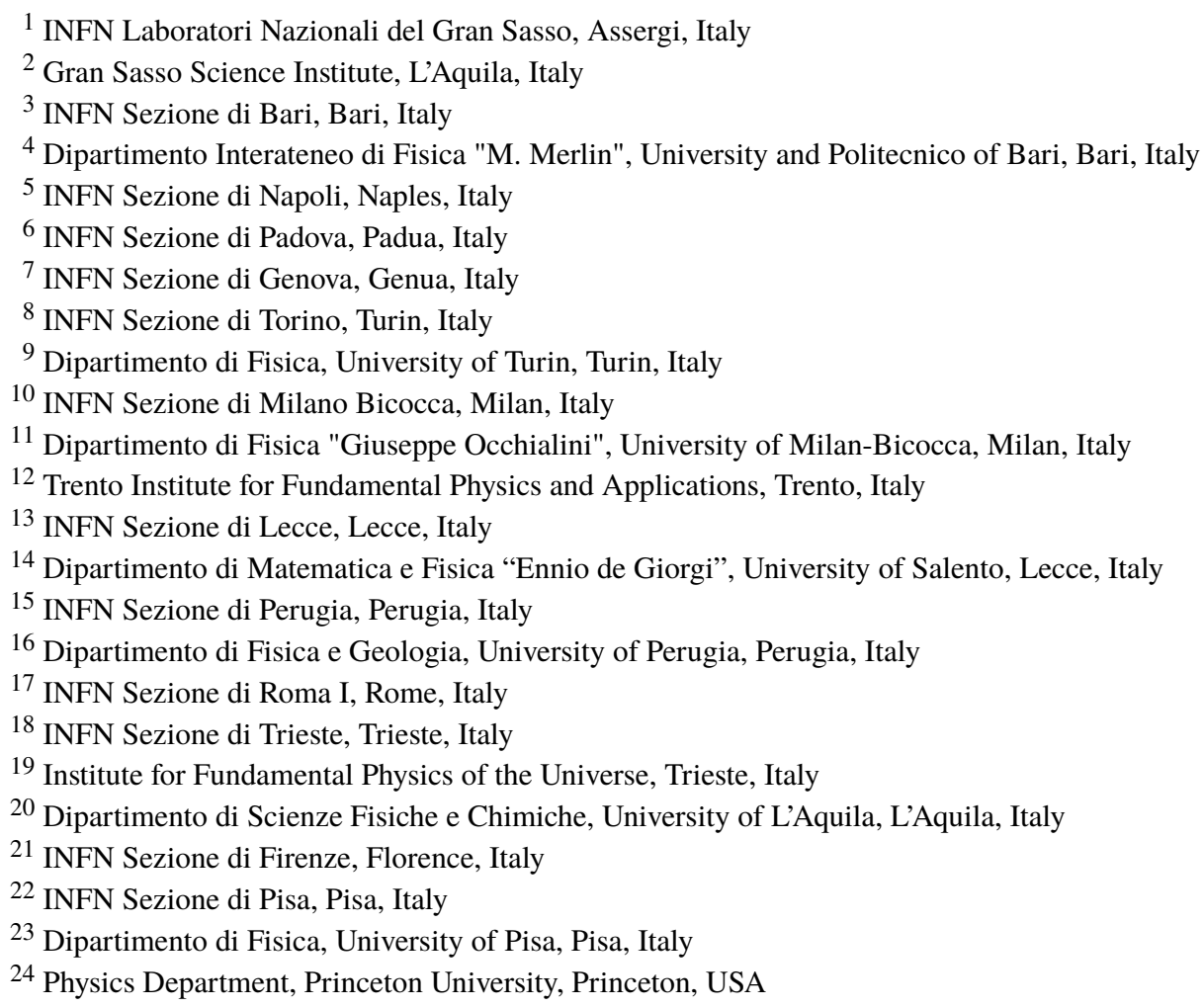


${ }^{25}$ INFN Sezione di Catania, Catania, Italy

${ }^{26}$ INFN Sezione di Milano, Milan, Italy

${ }^{27}$ Dipartimento di Fisica, University La Sapienza, Rome, Italy

${ }^{28}$ Dipartimento di Fisica e Astronomia "E. Majorana", University of Catania, Catania, Italy

${ }^{29}$ Dipartimento di Fisica "Ettore Pancini", University of Naples "Federico II", Naples, Italy

${ }^{30}$ Dipartimento di Fisica, University of Genua, Genua, Italy

${ }^{31}$ INFN Laboratori Nazionali del Sud, Catania, Italy

32 Dipartimento di Chimica e Farmacia, University of Sassari, Sassari, Italy

33 INFN Sezione di Roma Tor Vergata, Roma, Italy

34 Dipartimento di Fisica e Astronomia “G. Galilei”, University of Padua, Padua, Italy

35 INFN Laboratori Nazionali di Frascati, Gruppo Collegato di Cosenza, Cosenza, Italy

${ }^{36}$ Centro Ricerche Enrico Fermi, Rome, Italy

${ }^{37}$ Dipartimento di Fisica “A. Pontremoli”, University of Milan, Milan, Italy

${ }^{38}$ Liceo Patrizi, Cariati, Italy

${ }^{39}$ Dipartimento di Fisica, University of Trieste, Trieste, Italy

${ }^{40}$ CNR - Istituto di Scienze Applicate e Sistemi Intelligenti "Eduardo Caianiello", Pozzuoli, Italy

${ }^{41}$ Dipartimento di Fisica e Chimica "Emilio Segrè", University of Palermo, Palermo, Italy

42 INFN Sezione di Pavia, Pavia, Italy

${ }^{43}$ Dipartimento di Fisica, University of Pavia, Pavia, Italy

${ }^{44}$ Liceo Volta, Reggio Calabria, Italy

45 Centro di Ateneo di Studi e Attività Spaziali “G. Colombo", University of Padua, Padua, Italy

46 Dipartimento di Fisica, University of Calabria, Arcavacata di Rende, Italy

${ }^{47}$ Dipartimento di Matematica, University of Salerno, Salerno, Italy 